\title{
Determinacy of equilibrium outcome distributions for zero sum and common utility games
}

\author{
Cristian M. Litan ${ }^{\mathrm{a}}$, Francisco Marhuenda ${ }^{\mathrm{b}, *}$ \\ ${ }^{a}$ University Babeș-Bolyai, Faculty of Economics and Business Administration, Romania \\ ${ }^{\mathrm{b}}$ Department of Economics, University Carlos III, C/ Madrid 126, 28903-Getafe (Madrid), Spain
}

\author{
A B S T R A C T \\ We show the generic finiteness of probability distributions induced on outcomes by the Nash equilibria \\ in two player zero sum and common interest outcome games.
}

\footnotetext{
JEL classification:

C70
}

$\mathrm{C} 72$

Keywords:

Generic finiteness

Outcome zero sum games

Outcome common interest games

\section{Introduction}

It has been established by Harsanyi (1973) that for normal form games with an arbitrary number of players, if the payoffs of the players can be perturbed independently, generically there is a finite number of equilibria. Kreps and Wilson (1982) proved the generic finiteness of equilibrium probability distributions on the end nodes of an extensive form game. In games arising from economic models, it may happen that different actions of the players yield the same outcome. Thus, the scope of the above results is limited to the extent that it does not generally hold for outcome games.

Govindan and McLennan (2001) and Kukushkin et al. (2008) have shown examples for which there is a continuum of probability distributions on outcomes induced by the Nash equilibria of the associated game. A natural question that arises is to find out for

\footnotetext{
We thank an anonymous referee for his/her insightful comments.

虹 The authors acknowledge financial support from project No. ECO201019596 from the Spanish Ministry of Science and Innovation. Cristian Litan also acknowledges financial support by CNCSIS-UEFISCSU, project number PN II-RU $415 / 2010$

* Corresponding author. Tel.: +349162493 66; fax: +34916249329.

E-mail addresses: cristian.litan@econ.ubbcluj.ro (C.M. Litan), marhuend@eco.uc3m.es, marhuendf@gmail.com (F. Marhuenda).
}

which outcome games a similar result to the one obtained for normal and extensive form games continues to be valid.

Progress towards answering the above question has been made by Govindan and McLennan (2001) who have proved the generic finiteness of equilibrium distributions for games with two outcomes and any number of players. González-Pimienta (2010) argues that the same result holds for games with two players and three outcomes. Park (1997) has shown the generic determinacy of equilibria for sender-receiver cheap-talk games.

Mas-Colell (2010) has shown that the equilibrium payoffs are generically finite for two player game forms. In this note, we combine Mas-Colell's approach with elementary linear algebra to show the generic finiteness of equilibrium distributions on outcomes when the associated game is either a two player zero sum or a common interest game. This result has already been obtained, in a so far unpublished manuscript, by Govindan and McLennan (1998) using semi-algebraic geometry techniques. The interest of the present work lies on the fact that by relying only on straightforward linear algebra, we are able to provide a fairly short and elementary proof.

\section{Common utility and zero sum outcome games with two players}

There are two players. Let $S_{1}, S_{2}$ be their sets of pure strategies. We assume $n=\left|S_{1}\right| \geq 1, m=\left|S_{2}\right| \geq 1$ and let $S=S_{1} \times S_{2}$. 
Consider a finite set of outcomes $\Omega=\{1, \ldots, l\}$. We denote by $\Delta(\Omega)$ the set of probability measures on $\Omega$. An outcome game form is a function $\theta: S \rightarrow \Delta(\Omega)$.

For each outcome $j=1, \ldots, l$, the mapping $\theta$ defines a function $M^{j}: S \rightarrow \mathbb{R}$ such that $M^{j}\left(s_{a}, s_{b}\right)$ is the probability that $\theta\left(s_{a}, s_{b}\right)$ assigns to the outcome $j \in \Omega$. We identify each $M^{j}$ with an $n \times m$ matrix with real entries. Given $u=\left(u_{1}, \ldots, u_{l}\right) \in \mathbb{R}^{l}$, we interpret $u_{j}$ as the utility assigned to outcome $j$. To each $u \in \mathbb{R}^{l}$ we assign the matrix

$A(u)=\sum_{j=1}^{l} u_{j} M^{j}$

Given two profiles of utilities on outcomes $u^{1}, u^{2} \in \mathbb{R}^{l}$ for the players, the matrices $A\left(u^{1}\right)$ and $A\left(u^{2}\right)$ define a two-person game. The strategies $x \in \Delta\left(S_{1}\right)$ and $y \in \Delta\left(S_{2}\right)$ of the players induce a probability distribution on $\Omega$. The probability that outcome $j$ occurs is given by $x M^{j} y$.

A common utility game ${ }^{1}$ is a game in which $u=u^{1}=u^{2}$. We will write $A(u)$ for common utility games. The purpose of this work is to provide an elementary proof of the following result in Govindan and McLennan (1998).

Proposition 2.1. There is an open dense subset $G \subset \mathbb{R}^{l}$ such that, for every $u \in G$, the set of probability distributions on outcomes induced by the Nash equilibria of the game $A(u)$ is finite.

\section{Proof of Proposition 2.1}

The notation $d_{p}$ represents the vector $(1, \ldots, 1) \in \mathbb{R}^{p}$. The $n \times 1$ matrix $A\left(u^{1}\right) y$ is also interpreted as a vector in $\mathbb{R}^{n}$. In the expression $A\left(u^{1}\right) y$, we regard the vector $y \in \mathbb{R}^{m}$ as an $m \times 1$ matrix. Similarly, for $x A\left(u^{2}\right)$. The scalar product of $z, t \in \mathbb{R}^{p}$ is written as $z \cdot t$. By $A^{t}$ we denote the transpose of the matrix $A$.

The number of pure NE of a finite game is finite. By eliminating those strategies that are played with zero probability, it is enough to prove Proposition 2.1 for completely mixed NE. Given two utility profiles $u^{1}, u^{2} \in \mathbb{R}^{l}$, if a pair of completely mixed strategies, $x=$ $x\left(u^{2}\right) \in \Delta\left(S_{1}\right)$ and $y=y\left(u^{1}\right) \in \Delta\left(S_{2}\right)$, is Nash equilibrium (NE) of the game defined by $A\left(u^{1}\right)$ and $A\left(u^{2}\right)$, then, they constitute a solution of the following systems of linear equations

$\begin{array}{ll}A\left(u^{1}\right) y=\alpha d_{n}, & y \cdot d_{m}=1 \\ x A\left(u^{2}\right)=\beta d_{m}, & x \cdot d_{n}=1\end{array}$

for some $\alpha=\alpha\left(u^{1}\right) \in \mathbb{R}$ (the payoff of player 1 ) and $\beta=\beta\left(u^{2}\right) \in$ $\mathbb{R}$ (the payoff of player 2 ). The proof is based on a detailed analysis of the solutions of the above equations.

We will rely on the following fact shown by Mas-Colell (2010). Let $k=\max \left\{\operatorname{rank} A(u): u \in \mathbb{R}^{l}\right\}$. There is an open, dense subset $G$ of $\mathbb{R}^{l}$ such that the following hold.

(a) $\operatorname{rank} A(u)=k$, for every $u \in G$. After reordering, if necessary, the strategies of the players, we may write $A=A(u)$ as

$A=\left(\begin{array}{ll}B & C \\ D & E\end{array}\right)$

where $B=B(u)$ is a $k \times k$ matrix with $\operatorname{det} B \neq 0$.

(b) The functions rank $\left(A(u) \mid d_{n}\right)$ and rank $\left(A^{t}(u) \mid d_{m}\right)$ are constant on $G$.

\footnotetext{
1 Our method applies as well to games for which $u=u^{1}=t u^{2}$, with $t \in \mathbb{R} \backslash\{0\}$. In particular, taking $t=-1$, Proposition 2.1 holds also for two-person zero sum games
}

Let $k_{1}=\operatorname{rank}\left(A(u) \mid d_{n}\right)$ and $k_{2}=\operatorname{rank}\left(A^{t}(u) \mid d_{m}\right)$, for any $u \in G$. Note that $k_{1}, k_{2} \in\{k, k+1\}$. The argument will be carried out by considering the possible values of $k_{1}$ and $k_{2}$.

Case 1: $k_{1}=k_{2}=k$.

First, remark that $B^{-1}(u)$ is defined for all $u \in G$. In all the Lemmas that follow, it is assumed that $k_{1}=k_{2}=k$. Note also that the matrices below depend on $u$.

Remark 3.1. Since,

$k=\operatorname{rank} B=\operatorname{rank}\left(\begin{array}{ccc}B & C & d_{k} \\ D & E & d_{n-k}\end{array}\right)=\operatorname{rank}\left(\begin{array}{cc}B & C \\ D & E \\ d_{k} & d_{m-k}\end{array}\right)$

the remaining rows of the above matrices are a linear combination of its first $k$ rows. Therefore, there is an $(n-k) \times k$ matrix $H$ and a vector $z \in \mathbb{R}^{k}$ such that $H B=D, H C=E, H d_{k}=d_{n-k}, z B=d_{k}$ and $z C=d_{m-k}$. Hence, $H=D B^{-1}, z=d_{k} B^{-1}$. And we have that

$D B^{-1} C=E, \quad D B^{-1} d_{k}=d_{n-k}$,

$d_{k} B^{-1} C=d_{m-k}, \quad$ for every $u \in G$.

Fix now $\bar{u}^{1}, \bar{u}^{2} \in G$. From now on, we assume that the game defined by the matrices $A\left(\bar{u}^{1}\right)$ and $A\left(\bar{u}^{2}\right)$ has a completely mixed NE. Later, we will consider the particular case of a common utility game, $\bar{u}^{1}=\bar{u}^{2}=\bar{u}$. However, this last assumption is not needed in the lemmas below.

Lemma 3.2. There are open subsets $U_{1}, U_{2}$ of $G$ such that $\bar{u}^{1} \in$ $U_{1}, \bar{u}^{2} \in U_{2}$ and $d_{k} B^{-1}(u) d_{k} \neq 0$, for every $u \in U_{1} \cup U_{2}$. Furthermore, for $u^{1} \in U_{1}$ and $u^{2} \in U_{2}$, the systems of linear equations (1) and (2) have a solution only for the following payoffs

$\alpha\left(u^{1}\right)=\frac{1}{d_{k} B^{-1}\left(u^{1}\right) d_{k}}, \quad \beta\left(u^{2}\right)=\frac{1}{d_{k} B^{-1}\left(u^{2}\right) d_{k}}$.

Proof. Let $\alpha$ be the payoff of player 1 in the completely mixed NE of the game $A\left(\bar{u}^{1}\right)$ and $A\left(\bar{u}^{2}\right)$. Since Eq. (1) has a solution, we have that

$\operatorname{rank}\left(\begin{array}{cc|c}B & C & \alpha d_{k} \\ D & E & \alpha d_{n-k} \\ d_{k} & d_{m-k} & 1\end{array}\right)=\operatorname{rank}\left(\begin{array}{c}A \\ d_{m}\end{array}\right)=k$

Therefore, the last row of the above augmented matrix is a linear combination of its first $k$ rows. There is a vector $z \in \mathbb{R}^{k}$ such that $z B=d_{k}$ and $\alpha z \cdot d_{k}=1$. Hence, $z=d_{k} B^{-1}$ and $\alpha z \cdot d_{k}=$ $\alpha d_{k} B^{-1} d_{k}=1$. It follows that $d_{k} B^{-1}\left(\bar{u}^{1}\right) d_{k} \neq 0$ and the payoff of player 1 is $\alpha\left(\bar{u}^{1}\right)$, where $\alpha$ is defined in Eq. (3) Consider the polynomial

$p(u)=\operatorname{det}(B(u))\left(d_{k} B^{-1}(u) d_{k}\right)$.

Since, $p\left(\bar{u}^{1}\right) \neq 0$, there is and open set $U_{1} \subset G$ such that $\bar{u}^{1} \in U_{1}$ and $p(u) \neq 0$ for every $u \in U_{1}$. In particular, $d_{k} B^{-1}(u) d_{k} \neq 0$ for every $u \in U_{1}$. Moreover, the above argument also shows that, for any $u \in U_{1}$, the only possible value of $\alpha$ for which the system of Eqs. (1) has a solution is $\alpha(u)$, as given in Eq. (3). A similar argument, using the payoff of player 2, determines the set $U_{2}$ and the value of $\beta$, the payoff of player 2 .

The following two lemma s restate the familiar procedure of writing the general solution of a linear system as a particular solution of the complete system plus the general solution of the associated homogeneous system. The novelty here is in showing that, near $\bar{u}^{1}$ and $\bar{u}^{2}$, we can express those solutions in a differentiable manner. 
Lemma 3.3. There are differentiable functions $\alpha: U_{1} \rightarrow \mathbb{R}, y_{p}:$ $U_{1} \rightarrow \mathbb{R}^{m}$ and $y_{h}: U_{1} \times \mathbb{R}^{m-k} \rightarrow \mathbb{R}^{m}$ such that for each $u \in U_{1}$,

(i) $A(u) y_{p}(u)=\alpha(u) d_{n}, d_{m} \cdot y_{p}(u)=1$.

(ii) $A(u) y_{h}(u, v)=0, d_{m} \cdot y_{h}(u, v)=0$, for every $v \in \mathbb{R}^{m-k}$.

Proof. We split $\mathbb{R}^{p}$ as $\mathbb{R}^{j} \times \mathbb{R}^{p-k}$. Accordingly, we will explicitly write the action of each of the block matrices $B, \ldots, E$ of $A$, when it acts on a vector in $\mathbb{R}^{n}$. For example, given $z=\left(z_{k}, z_{m-k}\right) \in \mathbb{R}^{m}=$ $\mathbb{R}^{m} \times \mathbb{R}^{m-k}$, we will write $A z=\left(B z_{k}+C z_{m-k}, D z_{k}+E z_{m-k}\right) \in$ $\mathbb{R}^{n} \times \mathbb{R}^{n-k}=\mathbb{R}^{n}$. Let $\alpha: U_{1} \rightarrow \mathbb{R}$ be given by Eq. (3). Define $y_{p}: U_{1} \rightarrow \mathbb{R}^{m}$ and $y_{h}: U_{1} \times \mathbb{R}^{m-k} \rightarrow \mathbb{R}^{m}$ as follows

$y_{p}(u)=\alpha(u)\left(B^{-1}(u) d_{k}, 0\right)$

$y_{h}(u, v)=\left(-B^{-1}(u) C(u) v, v\right)$.

Since, $\operatorname{det}(B(u)) \neq 0$ on $G$, all the above functions $\alpha, y_{p}$ and $y_{h}$ are differentiable. By Remark 3.1, it follows that

$A y_{p}=\alpha\left(B B^{-1} d_{k}, D B^{-1} d_{k}\right)=\alpha\left(d_{k}, d_{n-k}\right)=\alpha d_{n}$

$d_{m} \cdot y_{p}=\alpha d_{k} B^{-1} d_{k}=1$

and

$A y_{h}=\left(-B B^{-1} C v+C v,-D B^{-1} C v+E v\right)=0$

$d_{m} \cdot y_{h}=-d_{k} B^{-1} C v+d_{m-k} \cdot v=-d_{m-k} \cdot v+d_{m-k} \cdot v=0$.

Similarly, one can prove the following result.

Lemma 3.4. There are differentiable functions $\beta: U_{2} \rightarrow \mathbb{R}, x_{p}:$ $U_{2} \rightarrow \mathbb{R}^{n}$ and $x_{h}: U_{2} \times \mathbb{R}^{n-k} \rightarrow \mathbb{R}^{n}$ such that for each $u \in U_{2}$ we have,

(i) $x_{p}(u) A(u)=\beta(u) d_{m} d_{n} \cdot x_{p}(u)=1$.

(ii) $x_{h}(u, w) A(u)=0, d_{n} \cdot x_{h}(u, w)=0$, for every $w \in \mathbb{R}^{n-k}$.

Of course, not all the functions $y\left(u^{1}, v\right)=y_{p}\left(u^{1}\right)+y_{h}\left(u^{1}, v\right)$ and $x\left(u^{2}, w\right)=x_{p}\left(u^{2}\right)+x_{h}\left(u^{2}, w\right)$ obtained from the above lemma s correspond necessarily to a NE. For this, one would need to check also that the coordinates of $y\left(u^{1}, v\right)$ and $x\left(u^{2}, w\right)$ are nonnegative and that these strategies constitute a best response to the other player's action.

The proof of Proposition 2.1 for the case $k_{1}=k_{2}=k$ is now a straightforward application of Lemmas 3.3 and 3.4. Using the notation in those lemma s, we note first that, for $\left(u^{1}, u^{2}\right) \in U_{1} \times U_{2}$,

$x_{h}\left(u^{2}, w\right) A\left(u^{1}\right) y_{p}\left(u^{1}\right)=\alpha\left(u^{1}\right) x_{h}\left(u^{2}, w\right) \cdot d_{n}=0$.

Differentiating this expression with respect to $u_{j}^{1}$ we obtain that

$x_{h}\left(u^{2}, w\right)\left(M^{j} y_{p}\left(u^{1}\right)+A\left(u^{1}\right) \frac{\partial}{\partial u_{j}^{1}} y_{p}\left(u^{1}\right)\right)=0$.

We consider now the particular case of a common utility game in which $\bar{u}^{1}=\bar{u}^{2}=\bar{u}$. Since, $x_{h}(\bar{u}, w) A(\bar{u})=0$, when plugging $u^{1}=u^{2}=\bar{u}$ in the previous equation, it reduces to

$x_{h}(\bar{u}, w) M^{j} y_{p}(\bar{u})=0, \quad$ for every $w \in \mathbb{R}^{n-k}$.

Similarly, we see that $x_{p}(\bar{u}) M^{j} y_{h}(\bar{u}, v)=0, \quad$ for every $v \in \mathbb{R}^{m-k}$.

On the other hand, differentiating $A(u) y_{h}(u, v)=0$ with respect to $u_{j}$ and evaluating at $\bar{u}$, we see that

$0=M^{j} y_{h}(\bar{u}, v)+A(\bar{u}) \frac{\partial}{\partial u_{j}} y_{h}(\bar{u}, v)$.

Multiplying on the left by $x_{h}(\bar{u}, w)$, the second term vanishes. Thus, $0=x_{h}(\bar{u}, w) M^{j} y_{h}(\bar{u}, v) \quad$ for every $v \in \mathbb{R}^{m-k}$ and $w \in \mathbb{R}^{n-k}$.

Now, when player 1 follows the strategy $x(\bar{u}, w)$ and player 2 follows the strategy $y(\bar{u}, v)$, the probability that outcome $j$ occurs is

$$
\begin{aligned}
x(\bar{u}, w) M^{j} y(\bar{u}, v)= & x_{p}(\bar{u}) M^{j} y_{p}(\bar{u})+x_{p}(\bar{u}) M^{j} y_{h}(\bar{u}, v) \\
& +x_{h}(\bar{u}, w) M^{j} y_{p}(\bar{u})+x_{h}(\bar{u}, w) M^{j} y_{h}(\bar{u}, v) \\
= & x_{p}(\bar{u}) M^{j} y_{p}(\bar{u})
\end{aligned}
$$

which, for a fixed $\bar{u} \in G$, is independent of $v$ and $w$.

Case $2: k_{i}=k+1$ for some $i=1,2$.

Consider first the possibility that $k_{1}=k+1$. The case $k_{2}=$ $k+1$ is similar. Assume that for some $u^{1}, u^{2} \in G$, the game $A\left(u^{1}\right), A\left(u^{2}\right)$ has a NE in which player 1 uses completely mixed strategy. This player must be indifferent among all his strategies. Thus, the linear system (1) has a solution, for some $\alpha \in \mathbb{R}$. If $\alpha \neq 0$, then $d_{n}$ is a linear combination of the columns of $A$, but then $k_{1}=\operatorname{rank}\left(A(u) \mid d_{n}\right)=k$. Hence, $\alpha=0$.

Therefore, if $k_{1}=k+1$, the payoff of player 1 vanishes in any completely mixed NE of any game generated by utilities in $G$.

We claim that no common interest game generated by utilities in $\mathrm{G}$ can have a completely mixed NE. Otherwise, there is $\bar{u} \in G$ such that the game $A(\bar{u})$ has a completely mixed NE, say $x \in \Delta\left(S_{1}\right)$ and $y \in \Delta\left(S_{2}\right)$. In this equilibrium, the payoff of player 1 is zero. Since $G$ is open, we can add a small enough, non-zero constant $\varepsilon$ to the utilities of the players and obtain a new game, say $A(\bar{u}+\varepsilon)$, also generated by utilities from $G$. Since the payoffs of the games $A(\bar{u})$ and $A(\bar{u}+\varepsilon)$ differ only by a fixed constant $\varepsilon$, they have the same NE. In particular, $x \in \Delta\left(S_{1}\right)$ and $y \in \Delta\left(S_{2}\right)$ is also a completely mixed NE in the game $A(\bar{u}+\varepsilon)$, in which player 1 gets the payoff $\varepsilon$, but this contradicts the above remark.

\section{References}

González-Pimienta, C., 2010. Generic finiteness of outcome distributions for two person game forms with three outcomes. Mathematical Social Sciences 59, 364-365.

Govindan, S., McLennan, A., 1998. Generic finiteness of outcome distributions for two person game forms with zero sum and common interest utilities. Mimeo, University of Western Ontario.

Govindan, S., McLennan, A., 2001. On the generic finiteness of equilibrium outcome distributions in game forms. Econometrica 69, 455-471.

Harsanyi, J.C., 1973. Oddness of the number of equilibrium points: a new proof. International Journal of Game Theory 2, 235-250.

Kreps, D.M., Wilson, R., 1982. Sequential equilibria. Econometrica 50, 863-894.

Kukushkin, N.S., Litan, C., Marhuenda, F., 2008. On the generic finiteness of equilibrium outcome distributions in bimatrix game forms. Journal of Economic Theory 139, 392-395.

Mas-Colell, A., 2010. Generic finiteness of equilibrium payoffs for bimatrix games. Journal of Mathematical Economics 46, 382-383.

Park, I.U., 1997. Generic finiteness of equilibrium outcome distributions for sender-receiver cheap-talk games. Journal of Economic Theory 76, 431-448. 